\title{
SPECIFICS OF THE QUANTOMOBILE FORCE BALANCE
}

\author{
Jurij Kotikov \\ Saint Petersburg State University of Architecture and Civil Engineering \\ Vtoraja Krasnoarmejskaja st., 4, St. Petersburg, Russia \\ cotikov@mail.ru
}

Abstract

The article provides data on the concept of thrust forces' formation in a quantum engine (QE). Influence of difference in structural diagrams of vehicles with QEs and ICEs on their energy consumption is described. Functional differences in formation and control over thrust and speed characteristics of automobiles and quantomobiles are reviewed.

Differences in thrust balances of vehicles mentioned are analyzed. Possible topography of quantomobile regulatory characteristics is considered as well.

\section{Keywords}

Automobile, quantum engine, quantomobile, quantum thrust, force balance, regulatory characteristics.

\section{Introduction}

The opportunity of implementing non-fuel energy production ideas based on the use of the energy of the physical vacuum (Davies, 1985, Dirac, 1930, Fetta, 2014, McCulloch, 2014, Parker, 1991, Puthoff, 2010, Shawyer, 2006, Tajmar, 2018, Tesla, 2009 and others) seems more and more real. The Theory of Superunification proposed by Leonov V.S., and his quantum engine (QE) concepts formed based on that theory (Leonov, 2002, 2010a, 2010b, 2018) feature outstanding ideas and a harmonious structure.

The ability to draw energy from the global physical vacuum will result in a new technological paradigm involving the transport sector as well. Quantum engines will replace internal combustion and jet engines; the existing propulsion devices of vehicles will improve.

Previous studies of the author addressed basic provisions of the Theory of Superunification, described aspects of $\mathrm{QE}$ creation, predicted some features of automobiles with QEs - quantomobiles, reviewed possible stages of their studying, included a comparative computational analysis of thrust forces and energy consumption of the quantomobile and modern car (Kotikov, 2018a, 2018b, 2018c).

The present article continues expanding insights into the quantomobile concept, however, only hypothetically.
The article addresses issues of the quantomobile force balance and its control.

\section{Concept of thrust formation in quantum engines}

The technology of creating the force of artificial gravitation has already been implemented by Leonov V.S. in a number of trial designs of quantum (field) engines that generate a thrust impulse due to the interaction of QE operating elements with QST (quantized space-time) without the ejection of the reactive mass (Leonov, 2002, 2010a, 2010b, 2018).

The direction and value of the force vector $\mathbf{F}$ (thrust) generated by the QE is determined by the spatial gradient (grad) of the energy W (Leonov, 2010a):

$$
\mathbf{F}=\operatorname{grad} W=\nabla W=\frac{\partial W}{\partial x} \mathbf{i}+\frac{\partial W}{\partial y} \mathbf{j}+\frac{\partial W}{\partial z} \mathbf{k}
$$

where $\mathbf{i}, \mathbf{j}, \mathbf{k}$ are unit vectors along the $\mathrm{x}, \mathrm{y}, \mathrm{z}$ axes, respectively.

The modulus of force (1) is determined by the following expression (Leonov, 2018):

$$
\left|\operatorname{gradW}^{2}\right|=\sqrt{\left(\frac{\partial \mathrm{W}}{\partial \mathrm{x}}\right)^{2}+\left(\frac{\partial \mathrm{W}}{\partial \mathrm{y}}\right)^{2}+\left(\frac{\partial \mathrm{W}}{\partial \mathrm{z}}\right)^{2}}
$$


The direction of the unit gradient vector (force direction) $\mathbf{n}$ is determined by the ratio of function (1) to its modulus (2) (Leonov, 2018):

$$
\mathbf{n}=\frac{\operatorname{gradW}}{\left|\operatorname{gradW}^{2}\right|}=\frac{\frac{\partial \mathrm{W}}{\partial \mathrm{x}} \mathbf{i}+\frac{\partial \mathrm{W}}{\partial \mathrm{y}} \mathbf{j}+\frac{\partial \mathrm{W}}{\partial \mathrm{z}} \mathbf{k}}{\sqrt{\left(\frac{\partial \mathrm{W}}{\partial \mathrm{x}}\right)^{2}+\left(\frac{\partial \mathrm{W}}{\partial \mathrm{y}}\right)^{2}+\left(\frac{\partial \mathrm{W}}{\partial \mathrm{z}}\right)^{2}}}
$$

Equations (1)...(3) are valid for calculations of the force when energy diffuses in space unevenly and energy differentials are observed, and when the function of energy distribution in space $W=f(x, y, z)$ is known (Leonov, 2018). The Theory of Superunification provides a scientific basis for the creation of an artificial traction force (changing the direction of the force vector $\mathbf{n}$ ) and thrust $\mathbf{F}_{\mathrm{T}}$ (Figure 1).

Let us call the force $\mathbf{F}$ the "traction force" (denoting it as $\mathbf{F}_{\mathrm{T}}$ ) (in terms of its application to vehicle's structural elements) as well as the "thrust force" (in terms of the field response to its perturbations in the resonator (the term is borrowed from rocketry). In case the resonator is rigidly mounted onto the vehicle's body and there are no intermediate force transducers, both things will mean the same. However, if there are intermediate transducers (dampers, reduction gears, etc.), those forces may differ.

It should also be noted that the force (vector) can be decomposed into unit vectors:

$\mathbf{F}_{\mathrm{T}}=\mathbf{F}_{\mathrm{Tx}}+\mathbf{F}_{\mathrm{Ty}}+\mathbf{F}_{\mathrm{Tz}}$

The scalar form of this equation is as follows:

$$
\mathrm{F}_{\mathrm{T}}=\sqrt{\mathrm{F}_{\mathrm{Tx}}^{2}+\mathrm{F}_{\mathrm{Ty}}^{2}+\mathrm{F}_{\mathrm{Tz}}^{2}}
$$

Influence of difference in structural diagrams of vehicles with QEs and ICEs on their energy consumption

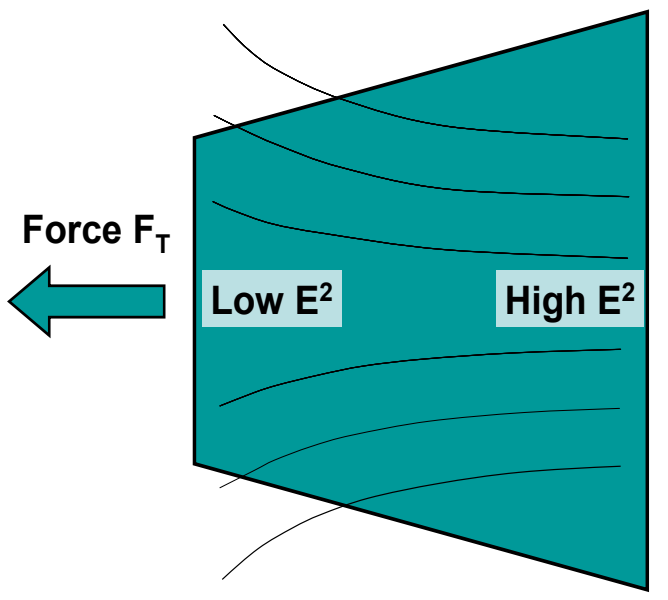

Figure 1. Gradient of field density in a QE conical resonator, resulting in thrust $F_{T}$ formation (Brandenburg, 2016)

To estimate quantomobile power characteristics compared to those of the modern automobile, the author considered a numerical example of comparing thrust and energy consumption by a KAMAZ-4326 truck (KAMAZmaster, 2018) and a hypothetical laboratory quantomobile based on such truck (in steady movement at a constant speed of $100 \mathrm{~km} / \mathrm{h}(27.8 \mathrm{~m} / \mathrm{s})$ ) (Kotikov, 2018c).

A difference structural diagram presented in Figure 2 was developed as a conceptual basis. The calculations accounted for features of the quantomobile passive wheeled running gear and improved aerodynamics under the vehicle body.

We will use the word "vehicle" as a common name for both the automobile and quantomobile.

Short excerpts from the study calculation results (Kotikov, 2018c) are presented in the following table. In that table, $G_{v e h}$ is the weight of a vehicle with a constant load for all five options: options 1 and 2 - gross vehicle weight, $10 \mathrm{t}$; options 3 and $4-$ weight reduction down to $8.8 \mathrm{t}$ due to removal of the transmission elements from the design; option 5 - weight reduction by another ton due to removal of the wheeled running gear elements from the design.
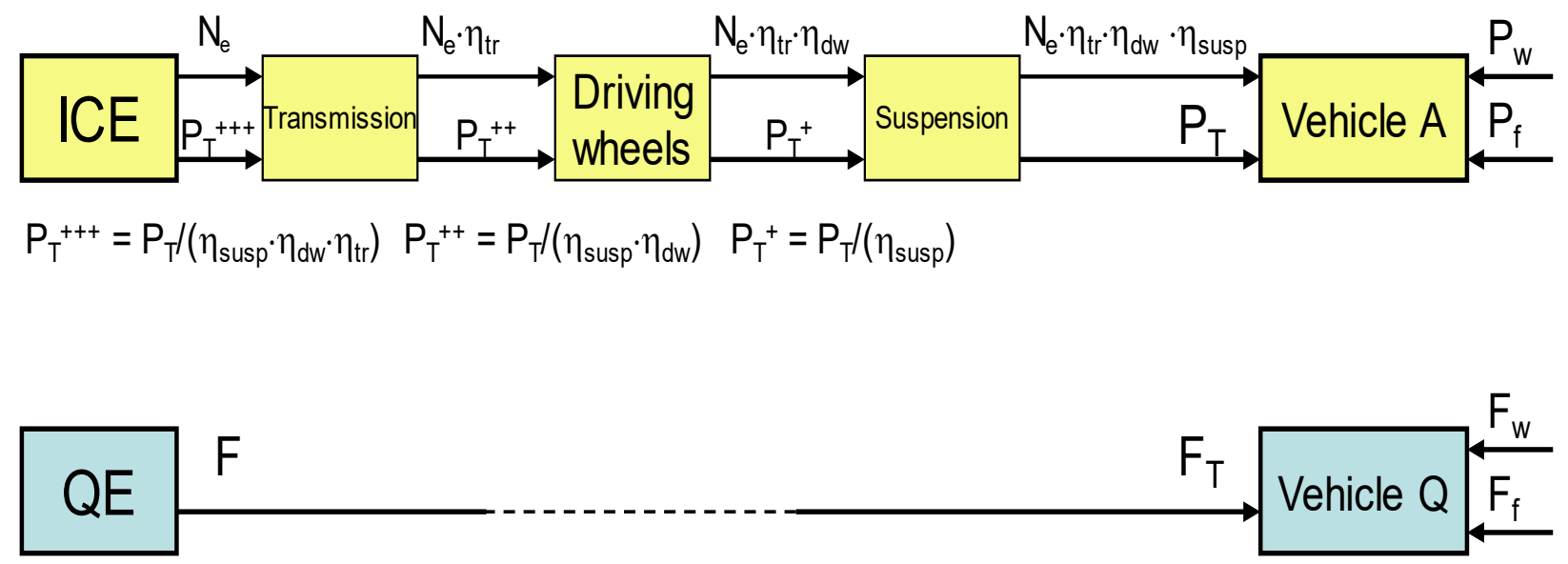

Figure 2. Diagrams of thrust generation for the automobile (Vehicle A) and quantomobile (Vehicle Q) (Kotikov, 2018c). 
Table 1. Comparative calculation results.

\begin{tabular}{|l|c|c|c|c|c|}
\hline \multirow{2}{*}{ Indicator } & \multicolumn{5}{|c|}{ Design cases } \\
\cline { 2 - 6 } & $\begin{array}{l}\text { 1. Automobile, ICE, } \\
\mathrm{f}=0.01, \mathrm{P}_{\mathrm{T}}\end{array}$ & $\begin{array}{l}\text { 2. Automobile, ICE, } \\
\mathrm{f}=0.1, \mathrm{P}_{\mathrm{T}}\end{array}$ & $\begin{array}{l}\text { 3. Quantomobile, } \\
\mathrm{QE}, \mathrm{f}=0.01, \mathrm{~F}_{\mathrm{T}}\end{array}$ & $\begin{array}{l}\text { 4. Quantomobile, } \\
\mathrm{QE}, \mathrm{f}=0.1, \mathrm{~F}_{\mathrm{T}}\end{array}$ & $\begin{array}{l}\text { 5. Flying car, QE } \\
\mathrm{F}_{\mathrm{Tx}}\end{array}$ \\
\hline $\mathrm{G}_{\mathrm{veh}}, \mathrm{N}$ & 100,000 & 100,000 & 88,000 & 88,000 & 78,000 \\
\hline $\mathrm{P}_{\mathrm{T}}\left(\mathrm{F}_{\mathrm{T}}, \mathrm{F}_{\mathrm{Tx}}\right), \mathrm{N}$ & 4,240 & 13,240 & 3,640 & 11,560 & 1,910 \\
\hline $\mathrm{N}_{\mathrm{T}}\left(\mathrm{N}_{\mathrm{Tx}}\right), \mathrm{kW} / \mathrm{hp}$ & $118 / 160$ & $368 / 500$ & $101 / 137$ & $321 / 437$ & $53 / 72$ \\
\hline $\mathrm{P}_{\mathrm{e}}+++\left(\mathrm{F}_{\mathrm{T}}, \mathrm{F}_{\mathrm{TX}}\right), \mathrm{N}$ & 5,730 & 22,830 & 3,640 & 11,560 & 1,910 \\
\hline $\mathrm{N}_{\mathrm{e}}\left(\mathrm{N}_{\mathrm{Tx}}\right), \mathrm{kW} / \mathrm{hp}$ & $159 / 216.5$ & $634 / 862$ & $101 / 137$ & $321 / 437$ & $53 / 72$ \\
\hline
\end{tabular}

It is apparent that energy consumption of a quantomobile with carrying wheels at a speed of $100 \mathrm{~km} / \mathrm{h}$ is $1.5-2$ times less than that of a KamAZ-4326 truck (see the $\mathrm{N}_{\mathrm{e}}\left(\mathrm{N}_{\mathrm{TX}}\right)$ row of the table). Energy consumption of the flying car for the horizontal component of thrust $\mathbf{F}_{\mathrm{Tx}}$ is three times less than that of the truck under the lowest possible resistance of the horizontal road $f=0.01$ (compare columns 1 and 5 of the table).

The absence of data on energy consumption for vertical suspension of the flying car anywhere (component $\mathbf{F}_{\mathrm{Tz}}$ of thrust (4)) made it impossible to compare the flying car with the truck in terms of total energy consumption during long-term movement under operating conditions (therefore, only the horizontal component of thrust $\mathbf{F}_{\mathrm{Tx}}$ was taken into account).

\section{Functional differences in formation and control} over thrust and speed characteristics of automobiles and quantomobiles

Let us use well-known information related to formation of force and power balance in an automobile with an ICE as basic data for consideration of the proposed differences. As far as it is possible, we will transfer the concepts and terminology of the automobile and ICE theories to the constructed conceptual field of the quantomobile. We will apply the multi-parameter approach proposed by SorokoNovitsky V.I. $(1935,1955)$ for ICEs, and by Jante A. (1958) for automobiles, which was also caught up by the author of the present paper (Kotikov, 1978, 1981, 1982a, 1982b, 2006).

The rate of energy consumption determines power of an automobile, a power unit, and is calculated as the product of the power parameter by the speed parameter. ICE power is determined by the product of the implemented engine torque $M_{e}$ by the shaft speed $n_{e}$ :

$$
N_{e}=M_{e} \times n_{e}
$$

Dependences of the measured torque $M_{e}$ (and other parameters: position of regulatory elements, fuel supply, etc.) on speed ne are speed characteristics. The dependence $M_{e}=f\left(n_{e}\right)$ at limiting positions of the regulator is called the External Speed Characteristic (ESC). In case the ICE speed limits are achieved, the ESC changes into the Limit Regulatory Characteristic (LRC). Speed characteristics measured at intermediate positions of the regulator are Partial Speed Characteristics.
Figure 3 presents a set of speed characteristics for the KAMAZ-740 diesel engine with a two-mode regulator, measured with the participation of the author (Kotikov, 1982a, 2006). If the values of the fuel injection pump rack position $h_{r}$ are placed on the orthogonal Z-axis (in the direction towards the reader), then we will obtain a three-dimensional representation of the dependence $h_{r}=f\left(M_{e} \times n_{e}\right)$.

It is possible to perform similar actions for fuel supply $q_{c}$ and other engine parameters. Thus, based on parameters $M_{e}$ (on the X-axis) and $n_{e}$ (on the Y-axis), the multi-parameter characteristic of the ICE is plotted, being its functional specification. Force, speed and power indicators develop kinematics and dynamics of vehicle motion by means of the transmission and wheeled running gear.

Those dependencies forming the basis for force, power and energy balance of the automobile and its power train are provided to state that they are not needed in case we use a QE. There is no kinematic link between the speed and load modes in the power unit.

In case of a QE, things are different, and, probably, easier: there is a thrust vector applied to the vehicle design, which is to be controlled (besides, most likely, there will be nothing else to control). However, as we will see below, control over the automobile thrust balance in the estimated field $\left(M_{e} \times n_{e}\right)$ can be compared with control over the thrust vector (thrust) in a QE in the estimated field $\left(F_{T} \times V_{q}\right)$.

As QEs have no speed mode in principle, the thrust balance equation remains the main means of calculation and basis for control:

$F_{T}=F_{f}+F_{i}+F_{v}+F_{j}=F_{\psi}+F_{v}+F_{j}$

Analytical capabilities of equation (6) are graphically presented in Figure 4 that shows a comparison for the automobile (in black) and quantomobile that inherited its properties (weight, shape, etc.) (in blue).

In case of force calculations, driving conditions in terms of the clutch are usually limited by the $F_{\text {Tmax }}$ force at $\varphi=0.8$ (Selifonov, 2007); besides, the GG hyperbola enveloping the thrust force diagrams (corresponding to transmission speeds) is plotted from above, from the level of $\varphi=0.8$ In case of a quantomobile, acceleration is not limited by the wheel grip. Figure 4 shows the force limit as the $F_{\text {Tmax }}$ constant force (at $\varphi=1$ ), which corresponds to $1 \mathrm{~g}$ acceleration at the initial acceleration rate. 


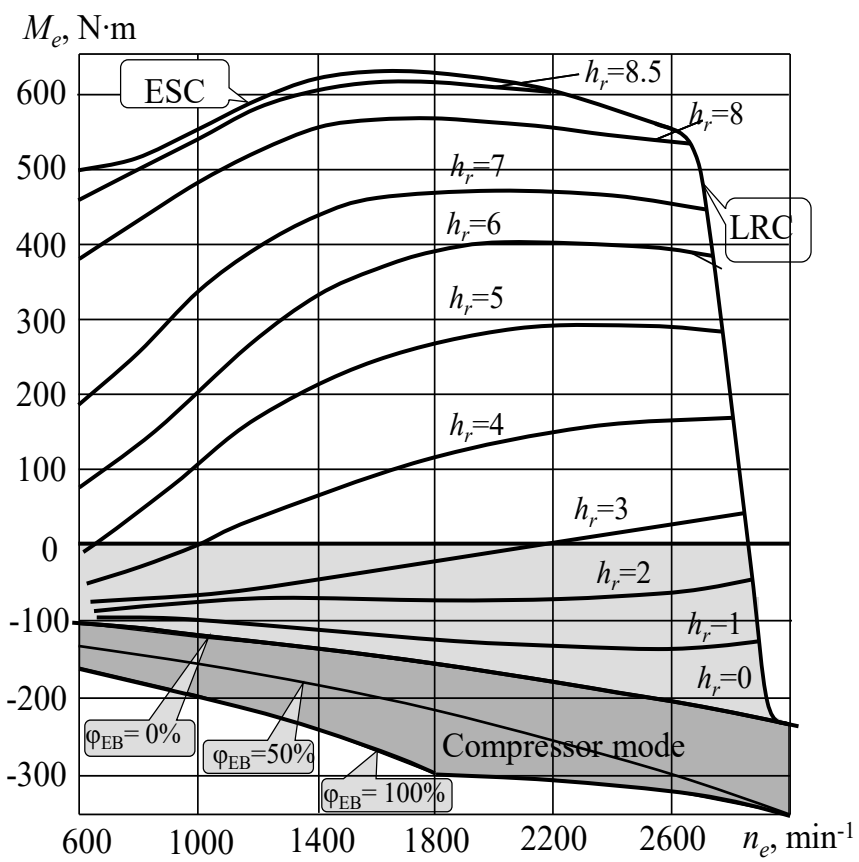

Figure 3. Set of speed characteristics $M_{e}=M_{e}\left(n_{e}\right)$ of the KamAZ-740 engine according to the position of the fuel injection pump rack $h_{r}$ (in mm), $\varphi_{\mathrm{EB}}$ - positions of the engine brake valve (Kotikov, 2006).

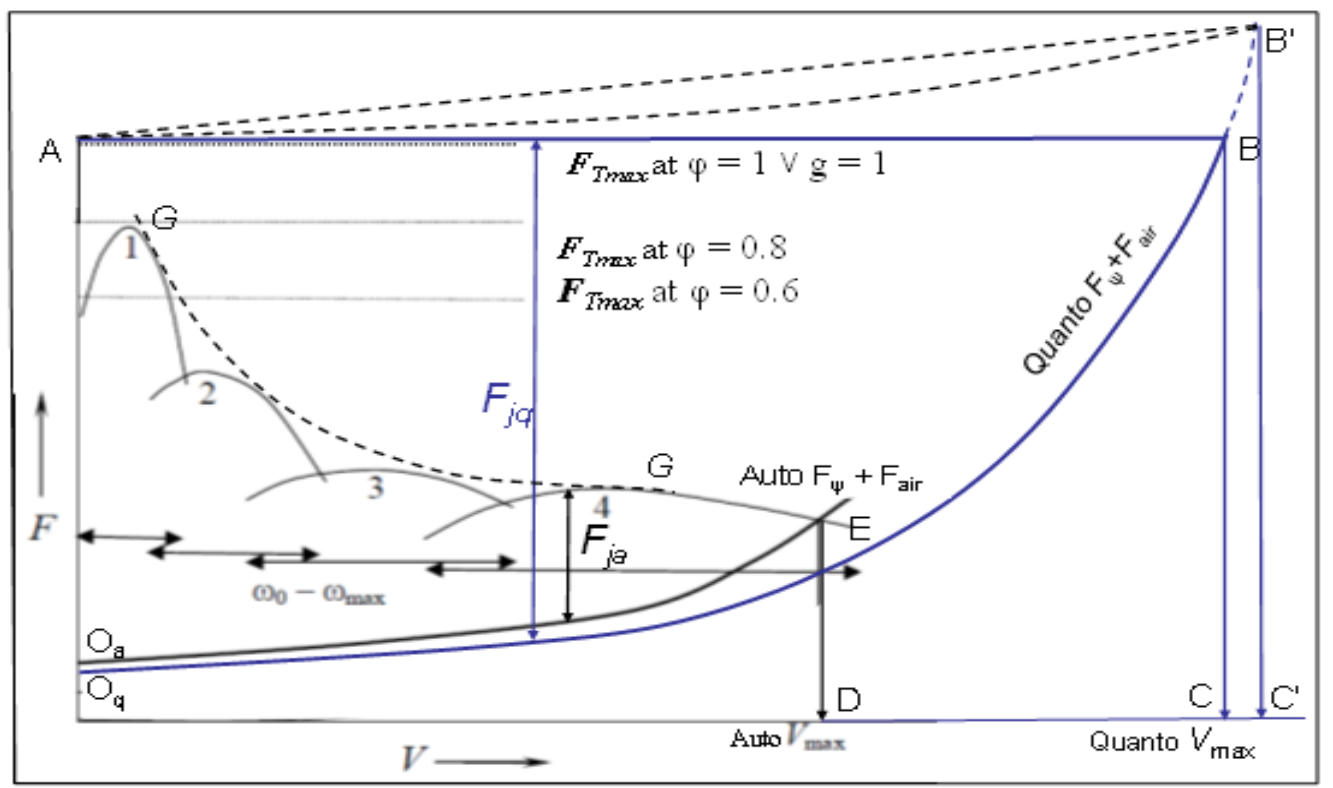

Figure 4. Thrust balance of the automobile and quantomobile.

It is obvious that the automobile has the remainder of the thrust force $F_{j a}$ for the dynamics, whereas the value of the $F_{i q}$ component in the quantomobile is several times larger than $F_{j a}$.

Moreover, lower consumption of the thrust force in a quantomobile for wheels' rolling and overcoming air resistance $\left(F_{\psi}+F_{\text {air }}\right)$ (see $\mathrm{O}_{\mathrm{a}} \mathrm{E}$ и $\mathrm{O}_{\mathrm{q}} \mathrm{B}$ curves), as well as the $F_{T \max }$ constant force (see blue $A B$ horizontal) result in the maximum acceleration rate of quantomobile (X-coordinate C of the contact point of curves B) which is much higher than the maximum automobile speed (compare points $C$ and D).
Even greater acceleration speed $C^{\prime}$ can be achieved by increasing the thrust force (controlled thrust $F_{T}$ ) of the QE according to the rectilinear or curvilinear law during acceleration (see lines $A B^{\prime}$ ). Thus, the two-channel (force and speed) multi-stage (chain: fuel injection pump regulator - fuel injection pump - combustion chamber clutch coupling - gearbox - main gear - final reduction gears - driving wheels) system converting driver's control actions into the thrust force is replaced in a quantomobile by single-channel control over intensity of operating unit (resonator) operation in a QE producing the thrust force (thrust) that is directly applied to the vehicle body. 


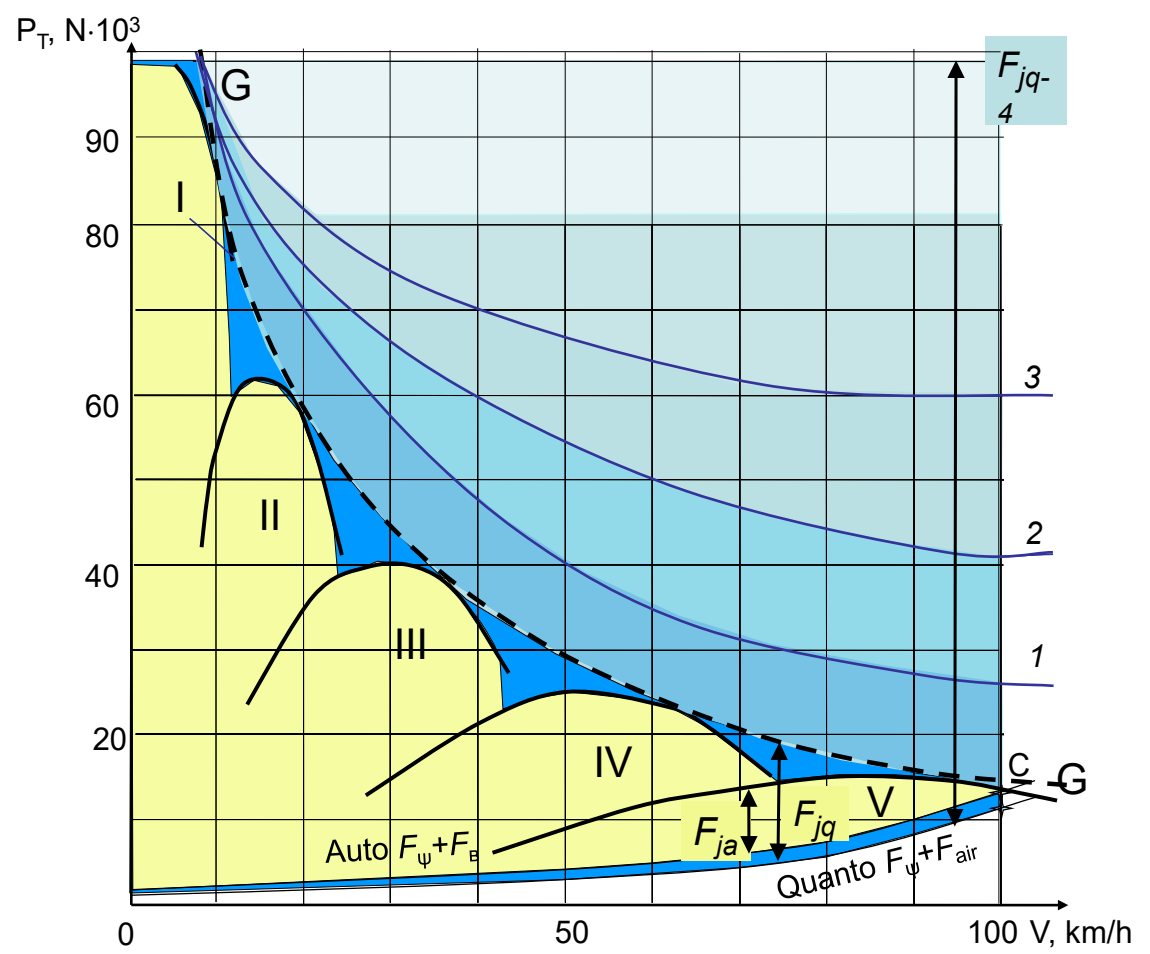

Figure 5. Thrust balance of a hypothetical automobile (yellow) and quantomobile that inherited its properties (blue) for $f=0.1$ : $G G-a$ hyperbola enveloping the thrust force diagrams corresponding to transmission speeds; curves 1, 2, 3, 4- hypothetical regulatory characteristics of a QE.

In terms of limit capabilities, the External Speed Characteristic (ESC) of the automobile ICE is replaced by the Limit Regulatory Characteristic (LRC) of the QE, which is represented by the dependence between the $\mathrm{F}$ scalar of the $\mathbf{F}_{\mathrm{T}}$ thrust and the limit (in terms of biomechanics or due to design constraints of a controlled or autonomous vehicle) vehicle acceleration. The LRC shall be set in accordance with the current vehicle weight (rigid LRC setting in accordance with the gross vehicle weight may lead to unacceptable accelerations of the unloaded vehicle).

An overview of the thrust balance of a hypothetical automobile in the speed range up to $100 \mathrm{~km} / \mathrm{h}$ is presented in Figure 5. The area of the automobile's thrust forces is highlighted in yellow. Transmission gear curves correspond to the maximum thrust efforts. Let us focus on the $F_{j a}$ and $F_{j q}$ forces representing the remainder of the thrust force for acceleration implementation in the automobile and quantomobile, respectively. As we can see, $F_{j q}>F_{j a}$. This can be confirmed analytically by the following equation for vehicle acceleration $a$ :

$a=(D-\psi) \frac{g}{\delta}$,

where $D-$ a vehicle dynamic factor;

$\psi$ - a road resistance coefficient;

$\delta-$ a coefficient to account for inertia of the vehicle's rotating masses.

The dynamic factor $D=\left(F_{T}-F_{\text {air }}\right) / G_{\text {veh }}$ of the quantomobile is higher as its air resistance $F_{\text {air }}$ is lower, and its total weight $G_{v e h}$ is lower as well. The coefficient $\psi$ for the quantomobile is also lower (due to the absence of the wheels' driving mode).
For the automobile:

$\delta=1+\delta_{1} \times i_{t r}{ }^{2}+\delta_{2}$,

where $\delta_{1}-$ a coefficient to account for inertia of the engine and transmission rotating masses, whereas $\delta_{2}$ a coefficient to account for inertia of the rotating wheels. Since the quantomobile has no engine and transmission rotating masses, in this case the equation term $\delta_{1} \times i_{t r}{ }^{2}$ drops out; besides, the $\delta$ value in equation (7) is always lower for the quantomobile than that for the automobile.

Thus, several factors work for higher acceleration values in the quantomobile, as compared to the prototype automobile. The dotted enveloping GG curve represents the geometric locus of the maximum thrust forces upon arbitrary variation of the series of transmission ratios of possible automobile gearboxes; this also reflects an option of using a variator with continuous changes in the transmission ratio.

If we consider the enveloping GG curve as a regulatory characteristic of quantomobile thrust, we will obtain an option of quantomobile movement with the structural load equivalent to that of the prototype automobile. Let us now focus on the $F_{j q}$ force. It is larger than the $F_{j a}$ force (mainly due to the lower values of wheels' rolling and air resistance). That means that the quantomobile can move with greater acceleration as compared to the prototype automobile.

Hyperbolas 1, 2, and 3 can act as QE regulatory characteristics for implementation of acceleration values much higher than those of the prototype automobile. The hyperbolic nature of those curves corresponds to the tendency towards vehicle acceleration decrease at high 
speeds, i.e. implementation of "carefulness" in terms of traffic safety. It would be possible to select those based on the operation conditions. Straight line 4 represents the QE Limit Regulatory Characteristic corresponding to the QE limit capabilities (if thrust augmentation $A B$ ' is not introduced (see Figure 4)).

\section{Concerning topography of quantomobile regulatory characteristics}

It is hard to tell how sophisticated the QE $F_{T}$ thrust regulator can be. But one thing is already clear - the Basic Regulatory Characteristic/Curve (BRC) will inevitably exist, represented by a framework for converting driver's (or automatic machine's) control actions into implementable thrust values:

$L=f_{L}\left(F_{T}, V_{q}\right)$

where $L$ - position of the driver's control lever.

It is also possible to use reverse functional (and graphical) representation:

$F_{T}=f_{F}\left(V_{q}, L\right)$

The Basic option implies that there is no account for hysteresis, no regulators (PID-, LQR-, Fuzzi- or others), as well as correctors (direct and reverse ones used to remove rigidity in the angles of the regulatory characteristic).

Taking the regulatory characteristic of a centrifugal two-mode regulator (Figure 3 ) as a prototype for BRC graphical plotting, we will make similar plottings for the BRC (Figure 6a).

Let us outline features of those BRCs. The convex nature of the curves in the two-mode ICE regulator (see Figure 3) can be explained by the non-linear convex nature of the engine torque speed characteristics (which, in turn, depend on non-linear convexity related to the efficiency coefficient within the engine operation field).

As the $\mathrm{QE}$ conversion coefficient CC (substituting the efficiency coefficient for a QE) is independent of the quantomobile speed mode (and due to the absence of specific $Q E$ characteristics), the two-mode regulation nature can be represented in the BRC by a series of horizontal lines (blue lines).

The ICE compressor mode during engine braking is represented by a set of curves close to horizontal lines (see the lower part of Figure 3). The angles of curves' deviation from the horizontal and their nonlinearity can be explained by the dependence of resistances to air flow in intake and exhaust systems on the speed mode. Since QEs have no such dependence, it is reasonable to represent the braking BRCs with a series of horizontals (red lines).

The regulatory characteristics of the thrust and braking modes meet on the line $\left(F_{1}+F_{\text {air }}\right)$ (see Figure 5 ) as in the absence of thrust the vehicle begins to slow down precisely on this line. This line also represents the boundary between the thrust zone (light blue) and braking zone (pink). For the purposes of understanding and comparison, the area of thrust modes of the prototype
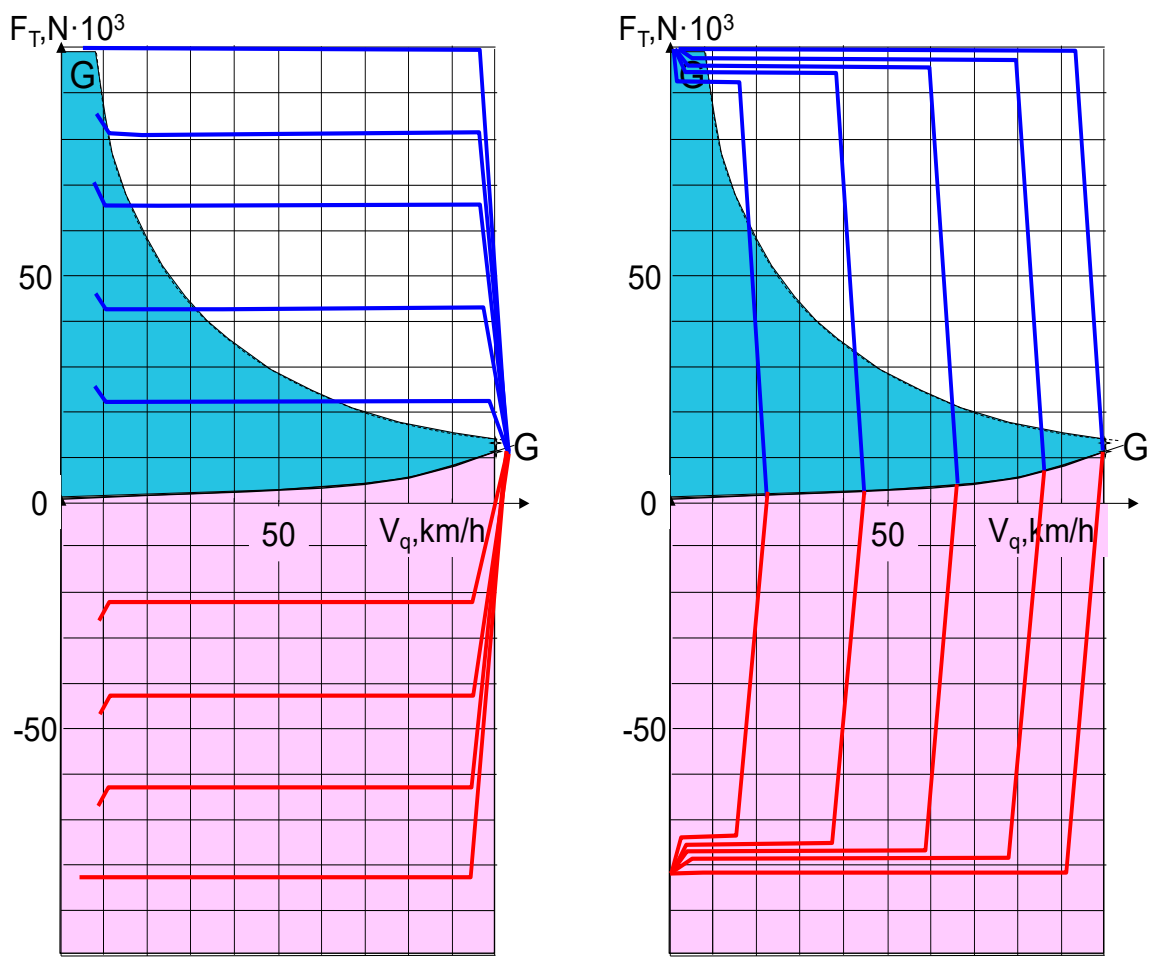

Figure 6. Basic regulatory characteristics of the quantomobile: a) two-mode; b) multi-mode 
automobile (transferred from Figure 5) is also marked with blue.

Figure $6 \mathrm{~b}$ shows the multi-mode BRC. This type of regulation is applicable for cruise control when it is necessary to maintain a relatively constant speed independent of changes in road resistance. Besides, in the absence of specific data on QEs (and at the start of their emergence), the curves are straight. The author provided for the mirror-like curves in the braking zone (pink) (in relation to the curves of the thrust zone (blue)). Most likely, in practice, there will be no such mirror reflection.
Moreover, combination of the two-mode and multi-mode topology of the set of curves for the same QE is possible.

\section{Conclusion}

Quantum engines and quantomobiles are still far from modern realities and capabilities. The key problems are associated with development of the physical vacuum.

The author believes that the present study approaches such development. Indeed, it is useful to evaluate capabilities of those fuzzy objects, as well as their hypothetical features and principle of control, in advance. 


\section{References}

Brandenburg, J. (2016). GEM theory of Q-V Thruster. Available at: http://ssi.org/wp-content/uploads/2017/02/ssi_estes_park_ proceedings_201609.pdf. (accessed on: 12.01.2019)

Davies, P. (1985). Superforce. New York: Touchstone.

Dirac P. (1930). The Principles of Quantum Mechanics. Oxford: The Clarendon Press.

Fetta, G. (2014). Electromagnetic Thruster. Patent US 2014/0013724 A1. Available at: http://www.rexresearch.com/fetta/ US2014013724A1.pdf (accessed on: 12.01.2019)

Jante, A. (1958). Mechanics of car movement, part 1. Moscow: Mashgiz.

Kotikov, J., Budyko, Yu., Lisitsyn, A. (1978). Use of modified universal characteristics for evaluation of fuel injection equipment of car engines. In: Proceedings of the Central Research \& Development and Design Institute of Fuel Injection Equipment for Automotive and Stationary Engines, 71, pp. 62-67.

Kotikov, J., Lukin A. (1981). Necessity to evaluate the automobile engine service features on its summarized and specialized service condition. Dvigatelestroyeniye, 3, pp. 45-47.

Kotikov, J.G., Gorev, A.E., Mamin, B.V., Shestakov, A.A. (1982a). Experimental evaluation of speed as well as fuel economy properties of a heavy-duty road train with two fuel injection pump options. Dvigatelestroyeniye, 10, pp. 46-47.

Kotikov, J.G. (1982b). Structure of the system for formation of estimates for performance of mobile machine engines. Dvigatelestroyeniye, 5, pp. 37-40.

Kotikov, J. (ed.) (2006). Transport power energy. Moscow: Academia Publishing Center.

Kotikov, J. (2018a). Structural properties and operational philosophy of the vehicle with the quantum engine. Architecture and Engineering, 3 (1), pp. 13-20. DOI: 10.23968/2500-0055-2018-3-1-13-20.

Kotikov J. (2018b). Stages of Quantomobile Development. Architecture and Engineering, 3 (2), pp. $26-35$. DOI: 10.23968/2500-0055-2018-3-2-26-35.

Kotikov J. (2018c). Comparative Analysis of Energy Consumption by Modern Cars and Future Quantomobiles. Architecture and Engineering, 3 (4), pp. 24-30. DOI: 10.23968/2500-0055-2018-3-4-26-32.

Leonov, V.S. (2002). Patent No. 2185526 (Russian Federation). A method of thrust generation in vacuum and a field engine for spaceship (options). Bulletin No. 20 dd. 20.07.2002 (priority date: 21.05.2001).

Leonov, V.S. (2010a). Quantum Energetics. Volume 1. Theory of Superunification. Cambridge: Cambridge International Science Publishing.

Leonov, V.S. (2010b). Space: Quantum Engine Test Results Regarding Thrust Generation Without Ejection. Available at: http:// www.astronomy.ru/forum/index.php/topic,77730.0.html (accessed on: 28.01.2018)

Leonov, V.S. (2018). Fundamentals of physics of a reactive thrust and nonreactive thrust]. Moscow: Amrita.

McCulloch, M. (2014). Physics from the Edge: A New Cosmological Model for Inertia. Singapore: Wspc.

Parker, B. (1991). Einstein's Dream. The Search for a Unified Theory of the Universe. (translated from English by V. I. Matsarskikh and O. I. Matsarskikh). Moscow: Nauka.

Puthoff, H. (2010). Advanced Space Propulsion Based on Vacuum (Spacetime Metric) Engineering. JBIS, 63, pp. 82-89.

Selifonov, V.V., Khusainov, A.Sh., Lomakin, V.V. (2007). Automobile theory: study guide. Moscow: Moscow State Technical University "MAMI".

Shawyer, R. (2006). A Theory of Microwave Propulsion for Spacecraft. Available at: https://www.newscientist.com/data/images/ ns/av/shawyertheory.pdf. (accessed on: 14.01.2019)

Soroko-Novitsky, V.I. (1935). Tests of automobile engines: fundamentals of the theory of measurement instruments, units for testing automobile engines, and mathematical processing of test results. Leningrad: Printing House named after Evgeniya Sokolova.

Soroko-Novitsky, V.I. (1955). Tests of automotive engines. Moscow: Mashgiz.

Tajmar, M., Kößling, M., Weikert, M., Monette, M. (2018). The SpaceDrive Project - First Results on EMDrive and Mach-Effect Thrusters. Available at: https://tu-dresden.de/ing/maschinenwesen/ilr/rfs/ressourcen/dateien/forschung/folder-2007-08-21-5231434330/ag_raumfahrtantriebe/SPC-The-SpaceDrive-Project-First-Results-on-EMDrive-and-Mach-EffectThrusters.pdf?lang=en. (accessed on: 14.01.2019)

Tesla, N. (2009). Patents. Samara: Agni Publishing House. 